\title{
Correlation between Local Structure Distortions and Martensitic Transformation in Ni-Mn-In alloys
}

\author{
D. N. Lobo ${ }^{1}$, K. R. Priolkar ${ }^{1}$, P. A. Bhobe ${ }^{2,3}$, D. Krishnamurthy ${ }^{4}$ and S. Emura ${ }^{4}$ \\ ${ }^{1}$ Department of Physics, Goa University, \\ Taleigao-Plateau, Goa-403206 India \\ ${ }^{2}$ Institute for Solid State Physics, The University of Tokyo, Kashiwa, Chiba 277-8581, Japan \\ ${ }^{3}$ RIKEN, SPring-8 Centre, Sayo-cho, \\ Sayo-gun, Hyogo 679-5148, Japan and \\ ${ }^{4}$ Institute of Scientific and Industrial Research, \\ Osaka University, 8-1 Mihogaoka, Ibaraki,Osaka 567-0047, Japan
}

(Dated: October 11, 2018)

\begin{abstract}
The local structural distortions arising as a consequence of increasing $\mathrm{Mn}$ content in $\mathrm{Ni}_{2} \mathrm{Mn}_{1+x} \operatorname{In}_{1-x}(\mathrm{x}=0,0.3,0.4,0.5$ and 0.6$)$ and its effect on martensitic transformation have been studied using Extended X-ray Absorption Fine Structure (EXAFS) spectroscopy. Using the room temperature EXAFS at the Ni and Mn K-edges in the above compositions, the changes associated with respect to the local structure of these absorbing atoms are compared. It is seen that in the alloys exhibiting martensitic transformation $(x \geq 0.4)$ there is a significant difference between the Ni-In and Ni-Mn bond lengths even in the austenitic phase indicating atomic volume to be the main factor in inducing martensitic transformation in Ni-Mn-In Heusler alloys.
\end{abstract}

PACS numbers: 
Non stoichiometric Ni-Mn-Z (Z = Sn and In) alloys exhibiting martensitic transformation in ferromagnetic state have been studied for their many novel properties. These alloys exhibit giant reverse magneto-caloric effect [1 7], large magnetic field induced strains [8 10], magnetic superelasticity [11] due to improved possibility of driving structural transformation by magnetic fields.

The martensitic transformation in these alloys has been a subject of debate in literature. Stoichiometric $\mathrm{Ni}_{2} \mathrm{MnIn}$ has a stable L2 1 crystal structure. Increasing substitution of In by Mn leads to martensitic instability with the transformation temperature $T_{M}$ steadily increasing with Mn concentration [12]. A simplistic explanation for such a behavior is the increase in electron per atom (e/a) ratio. However, this is not entirely convincing as $\mathrm{Ni}$ Mn-In alloys have higher $T_{M}$ than Ni-Mn-Sn alloys for a given Mn content inspite of the former having lower e/a ratio. Another interpretation that has emerged, after systematic studies on $\mathrm{Ni}_{2} \mathrm{Mn}_{1+x} \mathrm{Z}_{1-x}$ with $\mathrm{Z}=\mathrm{Ga}$, In, $\mathrm{Sn}$ and $\mathrm{Sb}$ is based on the atomic volume or the chemical pressure effect. According to this, the martensitic transformation occurs in alloys having austenitic lattice parameter, $a_{\text {cubic }} \approx 6.0 \AA$ or less [13]. This is important because the magneto-thermal and magneto-mechanical properties of these alloys have the same physical origin. The magnetic shape memory and magnetocaloric properties of these alloys are strongly connected to the martensitic transition. There exists an intimate relationship between structural and magnetic degrees of freedom in these alloys. Therefore there is a need to understand details of martensitic transformation to gain an insight of the magneto structural relationship and thereby into microscopic principles governing magnetic shape memory effect and magnetocaloric effect.

Recent EXAFS studies on $\mathrm{Ni}_{50} \mathrm{Mn}_{35} \mathrm{Sn}_{15}$ [14] and $\mathrm{Ni}_{50} \mathrm{Mn}_{35} \operatorname{In}_{15}$ [15] reveal a possibility of existence of local structural distortions even in the austenitic phase. However, in these studies there was no comparison made with stoichiometric alloys or compositions that do not undergo martensitic transformation. Therefore it is still not clear if the effect of Mn substitution leads only to a overall contraction of the lattice or there are some local structural distortions which play a more important role in inducing martensitic transformation.

In this letter, we focus our attention on the local structure around $\mathrm{Ni}$ and $\mathrm{Mn}$ in $\mathrm{Ni}_{2} \mathrm{Mn}_{1+x} \operatorname{In}_{1-x}(0 \leq x \leq 0.6)$ to gain a better understanding of microscopic effects responsible for martensitic transformation these magnetic shape memory alloys.

Polycrystalline $\mathrm{Ni}_{2} \mathrm{Mn}_{1+x} \mathrm{In}_{1-x}$ with $x=0,0.3,0.4,0.5$ and 0.6 were prepared by arc- 
TABLE I: Compositions, $\mathrm{T}_{c}$ and $\mathrm{T}_{M}$ of $\mathrm{Ni}_{2} \mathrm{Mn}_{1+x} \mathrm{In}_{1-x}$ alloys determined from EDX, magnetization and DSC measurements

\begin{tabular}{lccccc}
\hline Sample & Ni & Mn & In & $\mathrm{T}_{c}(\mathrm{~K}) \mathrm{T}_{M}(\mathrm{~K})$ \\
$\mathrm{Ni}_{2} \mathrm{MnIn}$ & 50.6 & 26.3 & 23.1 & 316 & - \\
$\mathrm{Ni}_{2} \mathrm{Mn}_{1.3} \mathrm{In}_{0.7}$ & 50.2 & 32.7 & 17.1 & 315 & - \\
$\mathrm{Ni}_{2} \mathrm{Mn}_{1.4} \mathrm{In}_{0.6}$ & 50.8 & 34.2 & 15.0 & 314 & 270 \\
$\mathrm{Ni}_{2} \mathrm{Mn}_{1.5} \mathrm{In}_{0.5}$ & 51.1 & 36.7 & 12.2 & - & 390 \\
$\mathrm{Ni}_{2} \mathrm{Mn}_{1.6} \mathrm{In}_{0.4}$ & 49.9 & 40.2 & 9.9 & - & 500 \\
\hline
\end{tabular}

melting the starting elements $(\geq 99.99 \%$ purity) under argon atmosphere. To ensure good homogeneity, the ingots were flipped over and re-melted 4-5 times with a total weight loss of $\leq 1 \%$ and annealed at the temperature of $1000 \mathrm{~K}$ for $48 \mathrm{~h}$ in an evacuated quartz ampoule followed by quenching in ice cold water. The samples were characterized X-ray diffraction, Energy dispersive analysis by X-rays (EDX), differential scanning calorimetry (DSC) and magnetization for their structure, composition and magnetic and martensitic properties. EXAFS at Ni K and Mn K edges were recorded at Photon Factory using beamline 12C at room temperature. For EXAFS measurements the samples to be used as absorbers, were ground to a fine powder and uniformly distributed on a scotch tape. These sample coated strips were adjusted in number such that the absorption edge jump gave $\Delta \mu t \leq 1$ where $\Delta \mu$ is the change in absorption coefficient at the absorption edge and $t$ is the thickness of the absorber. The incident and transmitted photon energies were simultaneously recorded using gas-ionization chambers as detectors. Measurements were carried out from $300 \mathrm{eV}$ below the edge energy to $1000 \mathrm{eV}$ above it with a $5 \mathrm{eV}$ step in the pre-edge region and $2.5 \mathrm{eV}$ step in the EXAFS region. At each edge, at least three scans were collected to average statistical noise. Data analysis was carried out using IFEFFIT [16] in ATHENA and ARTEMIS programs [17]. Here theoretical fitting standards were computed with FEFF6 [18, 19]. The data in the $\mathrm{k}$ range of $(2-12) \AA^{-1}$ and $\mathrm{R}$ range 1 to $3 \AA$ was used for analysis.

Ferromagnetic ordering temperature, $\mathrm{T}_{c}$ and $\mathrm{T}_{M}$ obtained from magnetization and DSC measurements for the alloys along with their compositions obtained from EDX are listed in Table I. These values agree well with those reported in literature [12].

The magnitude and real component of Fourier transform (FT) of the EXAFS spectra 
at the $\mathrm{Ni}$ K-edge for all $\mathrm{Ni}_{2} \mathrm{Mn}_{1+x} \mathrm{In}_{1-x}$ along with fitted curves are shown in Fig. 1, The spectra shown are uncorrected for phase difference, however the bond length values reported herein are phase corrected values. A dual peak structure is visible in the magnitude of FT in case of $\mathrm{Ni}_{2} \mathrm{MnIn}(x=0)$. This dual peak structure is due to backscattering from $\mathrm{Mn}$ and In atoms which surround the Ni atom. Since the phase difference introduced by the two atoms is different a dual peak structure results inspite of the two ( $\mathrm{Mn}$ and In) being equidistant from the central absorbing atom (Ni). With increasing Mn substitution in place of In $(x>0)$, the dual peak structure merges into a single broad peak.

In the austenitic phase, Ni occupies the body centered position in the B2 sub-cell of the L2 1 unit cell. Therefore Ni should be equidistant from Mn (Y-site) and In/Mn (Zsite). Indeed in case of $\mathrm{Ni}_{2} \mathrm{MnIn}, \mathrm{Ni}-\mathrm{Mn}$ and $\mathrm{Ni}-\mathrm{In}$ bond distances obtained from fitting Ni EXAFS are exactly equal. For all other compositions, differences between Ni-Mn and $\mathrm{Ni}$-In bond lengths are obtained with Ni-Mn bond distance being always shorter than NiIn bond distance. This is very clear from the bond length values reported in Table II. The alloys with $x=0$ and 0.3 do not undergo martensitic transformation while the $x=$ 0.4 alloy undergoes martensitic transformation below room temperature. Yet there is a difference between the Ni-Mn and Ni-In bond distances. Therefore, the distortions in the non-stoichiometric alloys are of local nature which are present even in the austenitic phase where the structure is ordered $\mathrm{L} 22_{1}$. Furthermore, the bond distances obtained from $\mathrm{Mn}$ K-edge EXAFS also support this point. The Mn-Ni bond distances are in agreement with Ni-Mn bond lengths obtained from Ni EXAFS. Secondly, the Mn-In/Mn bond distances are smaller than Ni-Ni bond distances obtained from Ni EXAFS in all compositions except $\mathrm{x}=$ 0 indicating the presence of local distortion around the Mn site. In L $2_{1}$ ordered structure, $\mathrm{Ni}-\mathrm{Ni}$ or Mn-In bond length should be half the unit cell length and hence should be equal. This is true in case of $\mathrm{Ni}_{2} \mathrm{MnIn}$ but in case of alloys having excess $\mathrm{Mn}$ composition the Mn-In/Mn bond lengths are shorter. Considering the fact the alloys have $\mathrm{L} 2_{1}$ structure, the above differences in bond distance would imply that the Mn occupying In site is displaced from its normal crystallographic site causing a local structural disorder.

The variation of bond distances as a function of $x$ is plotted in Fig. 2, It is clearly seen that with increasing Mn concentration the Ni-Mn or Mn-Ni bond distances decrease much more than Ni-In bond distances and there is a sudden contraction in Ni-Mn bond length for compositions that exhibit martensitic transformation. In all these compositions 
TABLE II: Bond distances obtained from analysis of Ni and Mn K edge EXAFS in $\mathrm{Ni}_{2} \mathrm{Mn}_{1+x} \operatorname{In}_{1-x}$ alloys. The numbers in parenthesis indicate uncertainty in the last digit.

\begin{tabular}{lcccccc}
\hline Sample & \multicolumn{3}{c}{ Ni EXAFS } & \multicolumn{3}{c}{ Mn EXAFS } \\
& \multicolumn{5}{c}{ Bondlength $(\AA)$} \\
& $\mathrm{Ni-Mn}$ & $\mathrm{Ni}-\mathrm{In}$ & $\mathrm{Ni}-\mathrm{Ni}$ & $\mathrm{Mn}-\mathrm{Ni}$ & $\mathrm{Mn}-\mathrm{In}$ & $\mathrm{Mn}-\mathrm{Mn}$ \\
\hline $\mathrm{Ni}{ }_{2} \mathrm{MnIn}$ & $2.626(3)$ & $2.626(3)$ & $3.04(1)$ & $2.618(5)$ & $3.02(1)$ & - \\
$\mathrm{Ni}_{2} \mathrm{Mn}_{1.3} \mathrm{In}_{0.7}$ & $2.596(4)$ & $2.620(3)$ & $3.03(1)$ & $2.592(4)$ & $2.94(2)$ & $2.91(2)$ \\
$\mathrm{Ni}_{2} \mathrm{Mn}_{1.4} \operatorname{In}_{0.6}$ & $2.565(3)$ & $2.620(3)$ & $3.02(1)$ & $2.562(3)$ & $2.89(1)$ & $2.93(1)$ \\
$\mathrm{Ni}_{2} \mathrm{Mn}_{1.5} \operatorname{In}_{0.5}$ & $2.562(5)$ & $2.602(4)$ & $3.01(1)$ & $2.565(5)$ & $2.88(1)$ & $2.93(2)$ \\
$\mathrm{Ni}_{2} \mathrm{Mn}_{1.6} \operatorname{In}_{0.4}$ & $2.557(4)$ & $2.600(3)$ & $3.00(1)$ & $2.574(4)$ & $3.03(1)$ & $2.95(1)$ \\
\hline
\end{tabular}

the Ni-Mn bond distance is of the order of $2.56 \AA$. It may be recalled that in $\mathrm{Ni}_{2} \mathrm{MnGa}$, $\mathrm{Ni}-\mathrm{Mn}$ bond distance is about $2.54 \AA[20]$. The closeness of these two values indicate that indeed chemical pressure is responsible for an alloy to undergo martensitic transformation. It may be recalled that In is much larger than Ga while Mn has approximately the same size. However, in these alloys, the Ni-Mn bond distance is the average of distance between $\mathrm{Ni}$ and $\mathrm{Mn}$ atoms occupying the $\mathrm{Y}$ site $\mathrm{Ni}-\mathrm{Mn}(\mathrm{Y})$ and $\mathrm{Ni}$ and $\mathrm{Mn}$ occupying the Z-sites, $\mathrm{Ni}-\mathrm{Mn}(\mathrm{Z})$. Since the crystal structure in the austenitic phase is $\mathrm{L} 2{ }_{1}$, the $\mathrm{Ni}-\mathrm{Mn}(\mathrm{Y})$ bond length is expected to be similar to Ni-In. Therefore, in these non-stoichiometric Ni-Mn-In alloys chemical pressure is exerted by the excess Mn ion which replaces In at the Z-site in L2 1 Heusler structure. The Mn ion being smaller in size compared to In, it is displaced (by $\sim 0.06 \AA$ ) from its crystallographic position closer to Ni. This will result in a stronger hybridization between $\mathrm{Ni}$ and $\mathrm{Mn}$. The band structure calculations on these alloys suggest that density of states (DOS) near Fermi level is dominated by Ni 3d and Mn 3d bands [21]. A stronger hybridization between $\mathrm{Ni}$ and Mn can result in increased DOS inducing the material to undergo martensitic transformation. The increase in Mn concentration results in higher in the chemical pressure and therefore higher martensitic transformation temperature.

In conclusion, the $\mathrm{Ni}$ and Mn K edge EXAFS show that Mn doped alloys a local structural distortion exists around the Mn atoms even in the austenitic phase. As a result of this distortion the substituted Mn atoms are displaced from their crystallographic positions closer to $\mathrm{Ni}$ giving rise to a $\mathrm{Ni}-\mathrm{Mn}$ hybridization which is responsible for inducing martensitic 
transformation in these Heusler alloys.

The work at Photon Factory was performed under the Proposal No. 2009G214. Two of us (KRP and DNL) are grateful to Prof A. K. Raychaudhuri and DST for travel funding. Financial assistance from CSIR, New Delhi under the project 03(1100)/07/EMR II is acknowledged. PAB would like to acknowledge JSPS for fellowship.

[1] T. Krenke, E. Duman, M. Acet, E. F. Wasserman, X. Moya, L. Mañosa and A. Planes, Nat. Mater. 4, 450 (2005).

[2] P. A. Bhobe, K. R. Priolkar and A. K. Nigam, Appl. Phys. Lett. 91, 242503 (2007).

[3] M. Khan, I. Dubenko, S. Stadler and N. Ali, J. Appl. Phys. 102, 113914 (2007).

[4] J. Du, Q. Zheng, W. J. Ren, W. J. Feng, X. G. Liu and Z. D. Zhang, J. Phys. D 40, 5523 (2007).

[5] J. Liu, N. Scheerbaum, J. Lyubina and O. Gutfleisch, Appl. Phys. Lett. 93, 102512 (2008).

[6] M. Khan, N. Ali and S. Stadler, J. Appl. Phys. 101, 053919 (2007).

[7] I. Dubenko, M. Khan, A. K. Pathak, B. R. Gautam, S. Stadler and N. Ali, J. Magn. Magn. Mater. 321, 754 (2009).

[8] K. Koyama, K. Watanabe, T. Kanomata, R. Kainuma, K. Oikawa and K. Ishida, Appl. Phys. Lett. 88132505 (2006).

[9] R. Kainuma, Y. Imano, W. Ito, Y. Sutou, H. Morito, S. Okamoto, O. Kitakami, K. Oikawa, A. Fujita, T. Kanomata, and K. Ishida, Nature (London) 439, 957 (2006).

[10] R. Kainuma, Y. Imano, W. Ito, H. Morito, Y. Sutou, K. Oikawa, A. Fujita, K. Ishida, S. Okamoto, O. Kitakami, and T. Kanomata, Appl. Phys. Lett. 88, 192513 (2006).

[11] T. Krenke, E. Duman, M. Acet, E. F. Wassermann, X. Moya, L. Mañosa, A. Planes, E. Suard and B. Ouladdiaf, Phys. Rev. B 75, 104414 (2007).

[12] T. Krenke, M. Acet, E. F. Wasserman, X. Moya, L. Mañosa and A. Planes, Phys. Rev. B 73, $174413(2006)$.

[13] A. Planes, L. Mañosa and M. Acet, J. Phys.: Condens. Matter 21, 233201 (2009).

[14] P. A. Bhobe, K. R. Priolkar and P. R. Sarode, J. Phys.: Condens. Matter 20, 015219 (2008).

[15] P. A. Bhobe, K. R. Priolkar and P. R. Sarode, J. Phys. D: Appl. Phys. 41, 045004 (2008).

[16] M. Newville, J. Synchrotron Radiat. 8, 322 (2001). 
[17] B. Ravel and M. Newville, J. Synchrotron Radiat. 12, 537 (2005).

[18] B. Ravel, J. Synchrotron Radiat. bf 8, 314 (2001).

[19] S. I. Zabinsky, J. J. Rehr, A. Ankudinov, R. C. Albers and M. J. Eller, Phys. Rev. B 52, 2995 (1995).

[20] P. A. Bhobe, K. R. Priolkar and P. R. Sarode, Phys. Rev. B 74, 224425 (2006).

[21] E. Şaşioğlu, L. M. Sandratskii and P. Bruno, Phys. Rev. B bf 70, 024427 (2004).

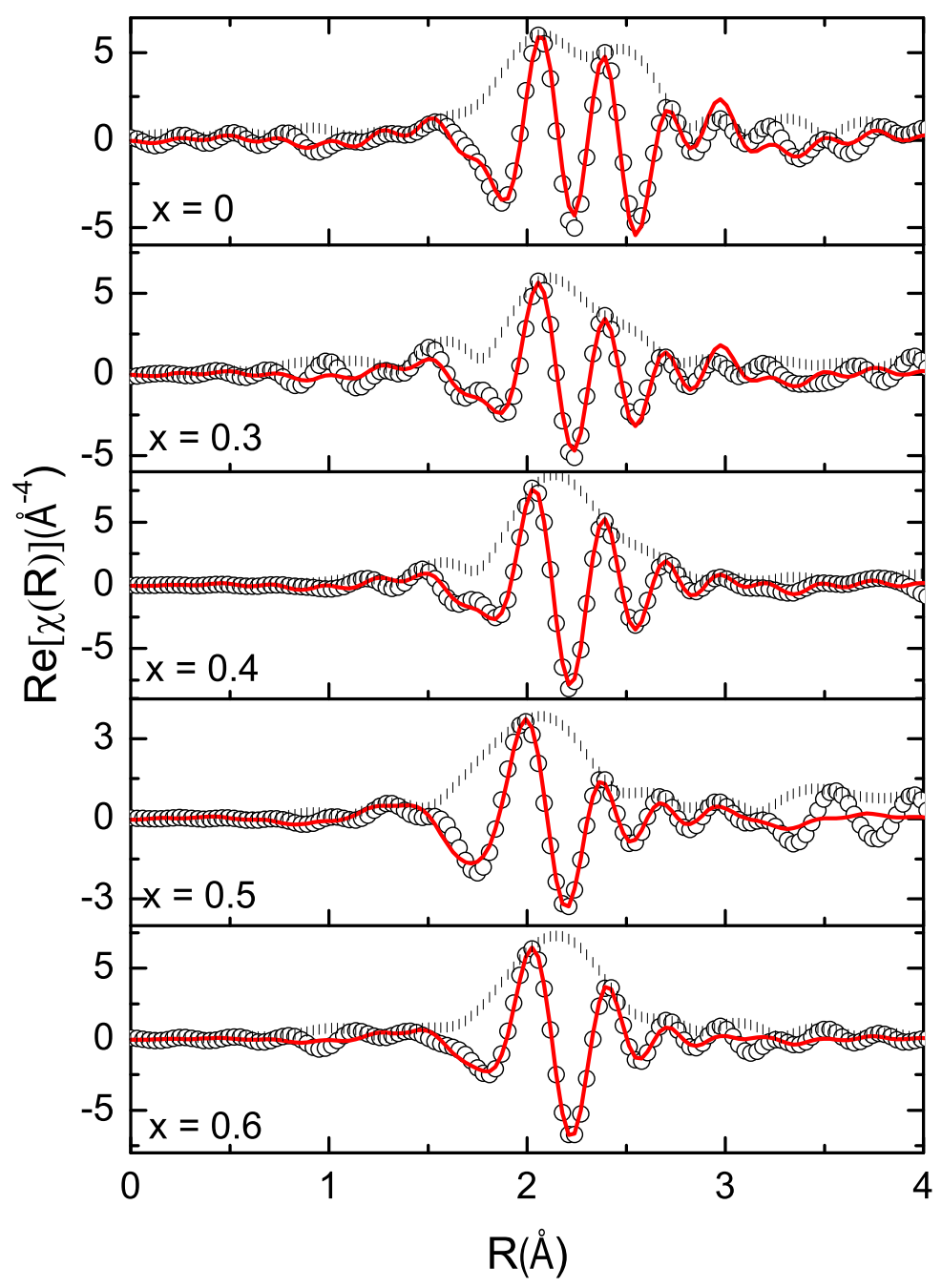

FIG. 1: Real part (circles) and magnitude (vertical lines) of Fourier transform of EXAFS spectra at the $\mathrm{Ni} \mathrm{K}$-edge in $\mathrm{Ni}_{2} \mathrm{Mn}_{1+x} \mathrm{In}_{1-x}$. The solid line is the best fit to the data. 


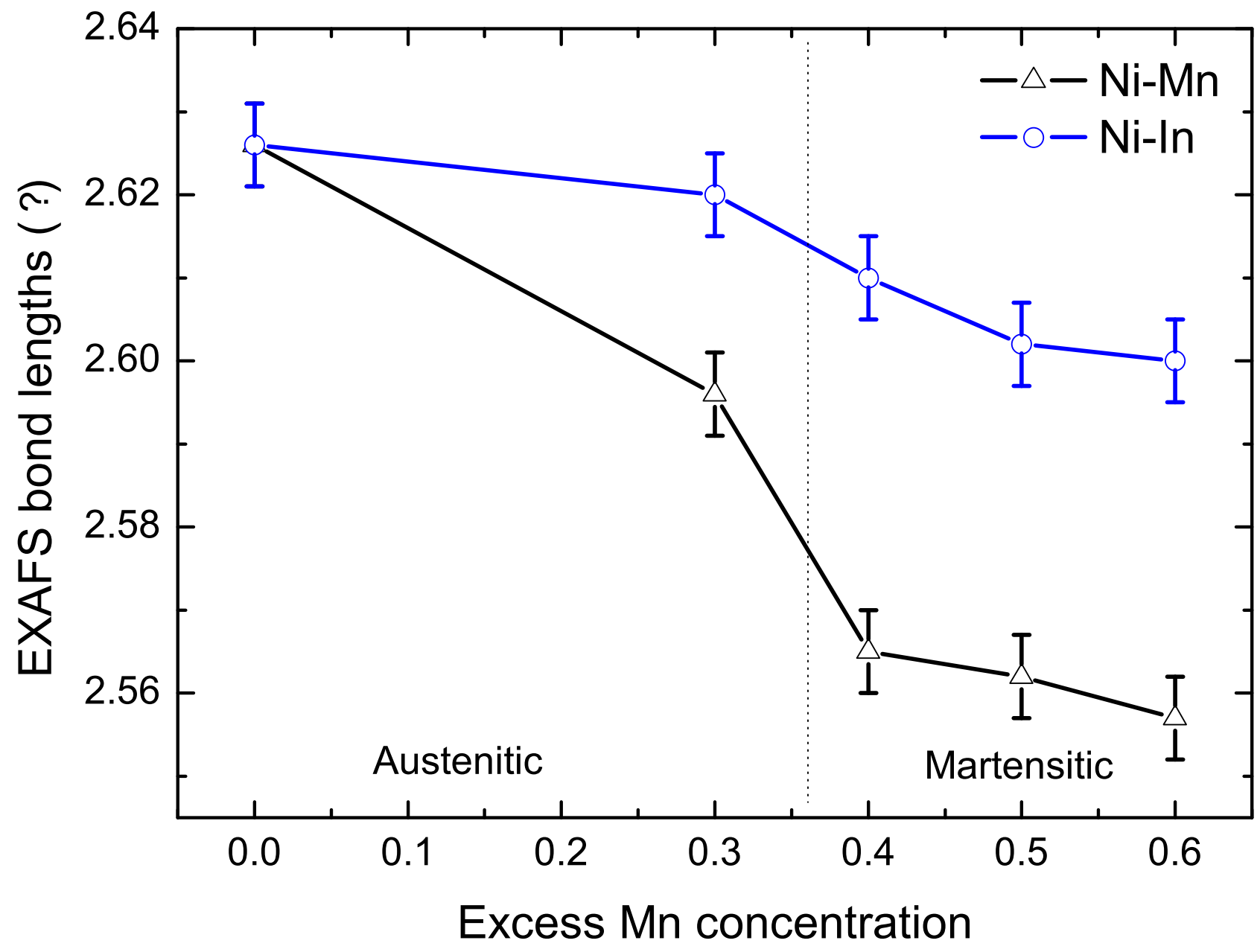

FIG. 2: Variation of Ni-Mn and Ni-In bond lengths obtained from EXAFS analysis as a function of excess $\mathrm{Mn}$ concentration in $\mathrm{Ni}_{2} \mathrm{Mn}_{1+x} \mathrm{In}_{1-x}$ alloys. 